\title{
Les effets du changement climatique sur la santé : Découvrez les risques et faites partie de la solution
}

\author{
C Howard ${ }^{1,2 *}$, P Huston $^{3}$
}

\section{Résumé}

Le changement climatique représente une menace claire et immédiate pour la santé humaine. Les répercussions sur la santé sont déjà observables au Canada, qui se réchauffe environ deux fois plus vite que la moyenne mondiale. Le Rapport sur l'écart entre les besoins et les perspectives en matière de réduction des émissions, récemment publié par les Nations Unies, indique que si les pays maintiennent leurs efforts actuels en matière d'émissions, les émissions dépasseront les objectifs fixés dans l'Accord de Paris et le réchauffement climatique dépassera $2{ }^{\circ} \mathrm{C}$ mondialement. L'augmentation des risques pour la santé est une conséquence importante du réchauffement planétaire. II est possible de prévenir et atténuer les effets des changements climatiques sur la santé, et l'identification et diffusion de ces stratégies constituent l'une des meilleures incitations à l'action. Cet éditorial présente un aperçu de certaines des initiatives mondiales et nationales en cours pour réduire les émissions et s'attaquer aux risques pour la santé du changement climatique en général, et met en lumière certaines des initiatives nationales en cours pour atténuer le risque accru de maladies infectieuses plus particulièrement au Canada.
Citation suggérée : Howard C, Huston P. Les effets du changement climatique sur la santé : Découvrez les risques et faites partie de la solution. Relevé des maladies transmissibles au Canada 2019;45(5):126-31. https://doi.org/10.14745/ccdr.v45i05a01f

Mots-clés : Changement climatique, effets sur la santé, maladies transmissibles, résilience, adaptation, prévention, émissions, éco-anxiété, deuil écologique, maladies transmises par les moustiques, surveillance
Cette oeuvre est mise à la disposition selon les termes de la licence internationale Creative Commons Attribution 4.0

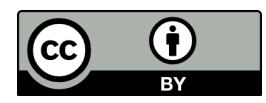

Affiliations

${ }^{1}$ Ministère de la Santé et des Services sociaux, Territoires du Nord-Ouest

${ }^{2}$ Canadian Association of Physicians for the Environment, Toronto, Ontario

${ }^{3}$ Direction générale de la prévention et du contrôle des maladies infectieuses, Agence de la santé publique du Canada, Ottawa, Ontario

${ }^{\star}$ Correspondance : courtghoward@gmail.com

\section{Introduction}

L'Organisation mondiale de la Santé a déclaré que « le changement climatique est le plus grand défi du $21^{\mathrm{e}}$ siècle, et menace tous les aspects de la société dans laquelle nous vivons » (1). Pour s'attaquer à ce défi, 175 pays ont ratifié l'Accord de Paris de la Convention-cadre des Nations Unies sur les changements climatiques (2). Collectivement, ces pays ont convenu de limiter l'augmentation moyenne de la température mondiale bien en deçà de $2{ }^{\circ} \mathrm{C}$, et de continuer les efforts pour limiter l'augmentation à $1,5^{\circ} \mathrm{C}$. Le Canada est un des signataires de l'Accord de Paris et s'est engagé à réduire les émissions de $30 \%$ des niveaux de 2005 d'ici 2030. Malheureusement, les émissions du Canada en 2016 ont en fait augmenté par rapport à 1990 (3). Bien que certains pays aient réalisé de grands progrès (notamment le Royaume-Uni et la Chine), le Rapport sur l'écart entre les besoins et les perspectives en matière de réduction des émissions de 2018 indique que si tous les pays maintiennent leurs efforts actuels en matière d'émissions, les émissions dépasseront les objectifs fixés dans l'Accord de Paris et le réchauffement planétaire dépassera $2{ }^{\circ} \mathrm{C}$ dans le monde (4).

Pour répondre au besoin de réduire les émissions et bâtir la résilience du climat, le gouvernement du Canada, après de vastes consultations avec les provinces, les territoires et les peuples autochtones, a élaboré un plan national sur les changements climatiques. Le Cadre pancanadien sur la croissance propre et les changements climatiques a été adopté par la plupart des premiers ministres canadiens en décembre 2016 (5). Le Cadre indique que les preuves sont claires : les activités humaines entraînent des changements sans précédent dans le climat de la Terre, qui créent des risques importants pour les communautés, la santé humaine, la sécurité, la croissance économique et l'environnement naturel. II indique que les effets des changements climatiques sont déjà évidents, car on a déjà documenté l'érosion côtière, le dégel du pergélisol, l'arrivée de maladies antérieurement confinées à des climats plus chauds, les augmentations des vagues de chaleur, des sécheresses et des inondations, de même que des risques aux infrastructures essentielles et à la sécurité alimentaire (5). Le plan pour remédier à cet état de choses au Canada s'articule autour de quatre piliers : tarification de la pollution par le carbone, mesures complémentaires pour réduire les émissions dans tous les secteurs, 
adaptation et résilience et technologies propres, innovation et emplois. Le Cadre comprend plus de 50 mesures et positions concrètes par lesquelles le Canada a l'intention de respecter les objectifs de réduction des gaz à effet de serre auxquels il s'est engagé dans l'Accord de Paris. Par exemple, des systèmes de tarification de la pollution par le carbone et des points de référence ont été établis partout au Canada.

Bien que les rapports annuels sur ce Cadre pancanadien aient documenté d'importants progrès (6), il existe encore un écart entre la situation actuelle et celle que nous devons atteindre pour que le Canada réalise ses objectifs. Le récent Rapport sur les changements climatiques au Canada de 2019 produit par Environnement et Changement climatique Canada, indique que le Canada se réchauffe deux fois plus vite que la moyenne mondiale, et que l'Arctique canadien se réchauffe en moyenne trois fois plus vite que le reste de la planète (7). Par exemple, la région du Delta McKenzie est déjà $3{ }^{\circ} \mathrm{C}$ plus chaude que dans les années 1950 (8).

\section{Les effets du changement climatique sur la santé}

Le respect de nos engagements sur les émissions n'est pas qu'une chose théoriquement souhaitable. Nous devenons de plus en plus conscients que les changements climatiques mènent à de nouvelles menaces pour la santé. Au plan international, des efforts tels que le Compte à rebours sur la santé et le changement climatique de Lancet indiquent clairement que certaines des plus importantes menaces à la santé à l'échelle mondiale incluent les effets néfastes liés à la chaleur sur la santé et la productivité au travail, l'aggravation des indicateurs de la sécurité alimentaire et l'intensification des répercussions des maladies transmissibles.

Au Canada, il existe de plus en plus de données probantes sur les menaces à la santé liées au climat. Avec la hausse des températures du nord, la sécurité alimentaire terrestre est menacée pour les populations autochtones $(9,10)$, la sécurité des déplacements sur la glace est réduite et la santé mentale est mise à rude épreuve (11). Les risques à la santé liés au climat partout au Canada comprennent une augmentation des coups de chaleur et de décès attribuables au nombre accru et à la durée des vagues de chaleur $(12,13)$; et les problèmes respiratoires causés par la pollution de l'air résultant des incendies de forêt (14). On s'attend à ce que les incendies de forêt extrêmes continuent à augmenter (7) entraînant des épisodes de pollution atmosphérique grave et des pressions accrues sur les établissements de santé et les prestataires de soins de santé (comme on l'a vu notamment lors de l'évacuation d'urgence de l'hôpital à Fort McMurray en 2016). Dans le même sens, on prévoit une augmentation des inondations, qui comportent leur propre risque de dommages à la propriété et de la perturbation personnelle et sociétale découlant de l'évacuation. Des symptômes d'anxiété et de trouble de stress post-traumatique ont été ressentis par des personnes affectées par des incendies, des inondations et des phénomènes météorologiques extrêmes (15). De nouvelles expressions telles que l'éco-anxiété et le deuil écologique font maintenant leur entrée dans le vocabulaire (16). Les risques de maladies transmissibles liées au changement climatique au Canada sont décrits dans le numéro d'avril 2019 du Relevé des maladies transmissibles au Canada et incluent une augmentation des maladies transmises par les tiques, (17) les maladies endémiques et exotiques transmises par les moustiques $(18,19)$ de même que les maladies d'origine alimentaire (20).

Il est démontré que la compréhension des répercussions des changements climatiques sur la santé est un des meilleurs moyens de motiver l'action (21), et les professionnels de la santé et les scientifiques sont parmi les messagers qui inspirent le plus confiance (22). Dans cet éditorial, nous mettons en lumière certaines initiatives nationales et mondiales récentes pour remédier au changement climatique et à ses effets sur la santé en général, et nous soulignons ensuite le travail lié plus particulièrement aux initiatives visant à minimiser les risques de maladies infectieuses émergentes.

\section{Initiatives mondiales}

Le récent Compte à rebours sur la santé et le changement climatique de Lancet indique que les professionnels de la santé et les systèmes de santé tiennent de plus en plus compte des effets du changement climatique sur la santé et y réagissent (23). Et pour cause : une meilleure compréhension des répercussions du changement climatique sur la santé permet d'améliorer la préparation, d'accroître la résilience et l'adaptation, et d'accorder priorité aux interventions d'atténuation qui protègent et favorisent le bien-être de l'être humain (23).

L'Organisation mondiale de la Santé travaille à un nouveau rapport intitulé Stratégie mondiale sur l'environnement, le changements climatiques et la santé (24). L'ébauche de Stratégie mondiale préconise une approche intégrée de la santé publique et de la science environnementale pour accélérer les travaux sur la prévention primaire et promouvoir des politiques qui s'attaquent aux causes premières des menaces de l'environnement pour la santé. Il est prévu que l'Assemblée de la Santé mondiale approuvera cette nouvelle stratégie en mai 2019.

L'adaptation consiste à réduire les effets associés à un niveau donné de changement climatique. En octobre 2018, on a annoncé une nouvelle Commission mondiale sur l'adaptation dirigée par l'ancien Secrétaire général des Nations Unies Ban Ki-moon, I'homme d'affaires américain Bill Gates et la directrice générale de la Banque mondiale, Kristalina Georgieva (25). Le rapport démontrera pourquoi l'adaptation aux risques climatiques est essentielle et décrira les actions nécessaires. La Commission présentera son rapport au Sommet sur le climat des Nations Unies en septembre 2019 à New York. 
Mondialement, il existe un mouvement de jeunes qui appelle à l'action sur le changement climatique lancé par une activiste suédoise de 16 ans, Greta Thunberg (26). Le 15 mars 2019, 1,5 million de jeunes et leurs supporteurs dans 123 pays ont manifesté partout dans le monde exigeant une réponse importante au changement climatique suivant une lettre ouverte aux dirigeants mondiaux qui déclarait : « Vous nous avez laissé tomber » (27). Rien qu'à Montréal, on a estimé la présence d'environ 150000 manifestants (28).

\section{Initiatives canadiennes}

Les effets du changement climatique sur la santé au Canada ne sont pas sans avoir été remarqués par les professionnels des soins de santé. En février 2019, I'Association médicale canadienne, l'Association des infirmières et des infirmiers du Canada, I'Association canadienne de santé publique, I'Association canadienne des médecins pour l'environnement et le Réseau pour la santé publique urbain ont lancé un appel à l'action, définissant le changement climatique comme une urgence sanitaire (29). La Fédération des étudiants et étudiantes en médecine du Canada et la Fédération internationale des associations d'étudiants en médecine ont toutes deux demandé que la santé climatique commence à être intégrée à l'enseignement médical d'ici la fin de 2020, et qu'elle le soit davantage d'ici 2025 (30). Sans cet enseignement, les professionnels de la santé ne seront pas préparés aux problèmes de santé liés au climat qu'ils sont susceptibles de voir fréquemment dans leurs pratiques au cours des années à venir.

Le Canada est en voie d'éliminer l'énergie au charbon d'ici 2030, une politique qui devrait produire 1,3 milliard de dollars en bénéfices sanitaires et environnementaux attribuables à une meilleure qualité de l'air (31). Le Canada a maintenant cofondé I'Alliance : Énergiser au-delà du charbon avec le Royaume-Uni dans le but de catalyser les efforts internationaux (32) qui pourraient multiplier ces avantages pour la santé. Dans le même sens, le nouveau Guide alimentaire du Canada, avec son accent sur une diète riche en plantes, est susceptible d'entraîner une réduction des émissions de gaz à effet de serre résultant de la production et de la consommation d'aliments composés de viande. Tout cela correspond aux recommandations de la EAT-Lancet Commission qui en décrit les avantages sanitaires tant pour l'être humain que pour la planète $(33,34)$.

De grands changements sont également possibles au niveau communautaire. En ce qui concerne le transport durable, les médecins et les praticiens de la santé publique encouragent davantage une infrastructure de transport actif dans de multiples collectivités pour réduire les taux d'émissions et améliorer la santé (35). L'engagement de Vancouver à devenir l'une des villes les plus durables au monde contient plusieurs éléments visant à augmenter les niveaux d'activités physique et diminuer la pollution (36). Faire sortir les gens des voitures alimentées par des combustibles fossiles et leur faire utiliser des modes de transport durables comme les bicyclettes et les autobus réduit non seulement les émissions, mais réduit également la pollution de l'air locale, améliore les niveaux d'activité et réduit les maladies.

\section{S'attaquer aux risques accrus de maladies in- fectieuses}

Dans ce numéro du Relevé des maladies transmissibles au Canada, vous lirez comment le gouvernement et les chercheurs universitaires du Canada ont trouvé des solutions pour remédier aux risques accrus de maladies transmissibles résultant du changement climatique. Un des principaux éléments s'est avéré être les stratégies de détection précoce et de prévention. Rees et al. décrivent une nouvelle génération de stratégies de surveillance pour la prédiction et la détection précoce d'éclosions de maladies transmissibles liées au climat, nommément la modélisation des risques et la surveillance basée sur des événements qui utilisent des données Internet de source ouverte, lesquelles ont récemment été améliorées par des applications d'intelligence artificielle, comme l'apprentissage automatique (37). Ogden et al. soulignent que comme le réchauffement, la variabilité climatique et les événements météorologiques extrêmes entraînent une augmentation de la fréquence et de l'intensité des maladies transmises par les moustiques, la compréhension des schémas météorologiques connexes peut faciliter la découverte précoce du moment où une région devient à risque. Des informations opportunes sur une éclosion imminente peuvent permettre la mise en œuvre de mesure de contrôle des moustiques et de communication du risque - avant que l'éclosion ne se produise (38). Kotchi et al. remarquent par ailleurs que les images de satellite peuvent fournir des données sur les indicateurs des déterminants environnementaux et climatiques qui influencent la présence et le développement des maladies transmises par les moustiques et les tiques. Ainsi, les données sur les changements de la température, de l'humidité, de la couverture végétale et bien plus encore peuvent servir à prédire des éclosions de maladies transmissibles. Des travaux sont désormais en cours pour augmenter la prévisibilité de cette technique en utilisant les données de multiples satellites et en appliquant des techniques novatrices d'apprentissage automatique pour traiter les mégadonnées (39). Germain et al. décrivent un nouveau modèle de collaboration entre les experts scientifiques et les décideurs de politiques publiques de la province de Québec dans une structure organisationnelle "Un monde, une santé » qui offre une plateforme pour le partage des connaissances, l'établissement de consensus et le développement de stratégies d'adaptation pour remédier au risque accru de maladies zoonotiques associées au changement climatique (40). 


\section{Discussions et conclusion}

Un sondage mené par lpsos-Reid en décembre 2018 a démontré que la majorité des Canadiens reconnaissent que le Canada a besoin de faire plus d'efforts pour s'attaquer au changement climatique (41). Cependant la sensibilisation sur les effets du changement climatique sur la santé n'est pas encore aussi étendue. II est important que tous les Canadiens sachent que le changement climatique affecte de plus en plus la santé de nombreuses manières. Les praticiens de soins de santé et de santé publique jouent un rôle important pour sensibiliser davantage le public sur ces risques à la santé liés au changement climatique. Un message positif peut être diffusé : une meilleure compréhension des effets du changement climatique sur la santé permet une meilleure préparation, une meilleure résilience et une meilleure adaptation, et l'élaboration de stratégies qui protègent et favorisent la santé humaine. Il y a beaucoup de travaux en cours pour remédier aux effets du changement climatique sur la santé dans le contexte de l'adaptation. Ces travaux sont axés sur la préparation, la prévention et la résilience. Ils comprennent une toute nouvelle génération de stratégies permettant de détecter quand nous courons un risque accru de maladies infectieuses liées au climat.

Les praticiens de soins de santé et de santé publique doivent être prêts à jouer un rôle actif dans la prévention, la détection précoce et l'atténuation des effets du changement climatique sur la santé. À cette fin, le contenu climat et santé doit être de plus en plus inclus dans les plans de cours et les cours de formation continue. Les effets nuisibles de la pollution de l'air et les effets traumatisants des phénomènes météorologiques extrêmes sont évidents et bien documentés. Par ailleurs, les effets du changement climatique sur les maladies transmissibles émergentes au Canada sont moins évidents et moins connus. Afin de ne pas rater l'apparition de maladies à transmission vectorielle induites par le changement climatique chez les personnes touchées, il faut de toute nécessité un indice élevé de suspicion et une confirmation en laboratoire. La compréhension et l'information opportune sur les nouvelles stratégies pour la détection précoce des risques et des éclosions de maladies émergentes susceptibles d'être induites par un changement climatique aideront les professionnels des soins de santé et de santé publique à se préparer et à réagir.

La connaissance des risques et le désir de faire partie des solutions sont des réactions saines au changement climatique. On fait beaucoup en ce moment et chaque professionnel des soins de santé peut faire beaucoup pour créer un avenir viable pour les générations actuelles et futures. Et cet effort en vaut la peine pour maintenir un état de préparation et de résilience individuelle, familiale et communautaire, pour contribuer à une chose plus grande que soi et aussi parce que le regard du monde est posé sur nous.

\section{Déclaration des auteurs}

Les deux auteurs ont élaboré la conception générale et la création de l'éditorial. $\mathrm{PH}$ a rédigé la première ébauche et $\mathrm{CH}$ et $\mathrm{PH}$ ont collaboré aux ébauches et aux révisions ultérieures.

$D^{\text {re }}$ Courtney Howard est présidente de l'Association des médecins pour l'environnement $\mathrm{D}^{\text {re }}$ Patricia Huston est rédactrice en chef du Rapport canadien sur les maladies transmissibles au Canada et elle s'est récusée de toute décision rédactionnelle relative au présent manuscrit. Les décisions ont été prises par le rédacteur invité du numéro d'avril 2019, le Dr Nicholas Ogden.

\section{Conflit d'intérêts}

Aucun. 


\section{Références}

1. Campbell-Lendrum D, Wheeler N, Maiero M, Villalobos Prats E, Nevelle T. World Health Organization COP24 Special Report on Health and Climate Change. World Health Organization; 2018. https://unfccc.int/sites/default/files/ resource/WHO\%20COP24\%20Special\%20Report_final.pdf

2. Nations Unies. Accord de Paris 2015. https://treaties.un.org/ doc/Treaties/2016/02/20160215\%2006-03\%20PM/Ch_XXVII7-d.pdf

3. Gouvernement du Canada. Émissions de gaz à effet de serre. 2018 (Accédé 2019-04-18). https://www.canada.ca/fr/ environnement-changement-climatique/services/indicateursenvironnementaux/emissions-gaz-effet-serre.html

4. United Nations Environment Programme. Emissions Gap Report 2018. http://wedocs.unep.org/bitstream/ handle/20.500.11822/26895/EGR2018_FullReport_ EN.pdf?sequence=1\&isAllowed =y

5. Gouvernement du Canada. Cadre Pancanadien sur la croissance propre et les changements climatiques. http:// publications.gc.ca/collections/collection_2017/eccc/En4294-2016-fra.pdf

6. Environnement et changement climatique Canada. Cadre pancanadien sur la croissance propre et les changements climatiques: Deuxième rapport annuel synthèse de la mise en oeuvre - Décembre 2018. Gouvernement du Canada. http://publications.gc.ca/collections/collection_2018/eccc/ En1-77-2018-fra.pdf

7. Bush E, Lemmen DS, editors. Rapport sur le climat changeant du Canada 2019; Gouvernement du Canada, Ottawa, ON. 446 p. https://www.rncan.gc.ca/sites/www. nrcan.gc.ca/files/energy/Climate-change/pdf/RCCC_ FULLREPORT-FR-FINAL.pdf

8. Government of the Northwest Territories. Climate Observations in the Northwest Territories (1957-2012) Inuvik * Norman Wells * Yellowknife * Fort Smith. Environment and Natural Resources. https://www.enr.gov.nt.ca/sites/enr/files/ page_3_nwt-climate-observations_06-13-2015_vf_1_0.pdf

9. Rosol R, Powell-Hellyer S, Chan HM. Impacts of decline harvest of country food on nutrient intake among Inuit in Arctic Canada: impact of climate change and possible adaptation plan. Int J Circumpolar Health 2016 Jul;75(1):31127. DOl PubMed

10. Berry P, Clarke K, Fleury MD, Parker S. "Santé humaine ", dans Vivre avec les changements climatiques au Canada : perspectives des secteurs relatives aux impacts et à I'adaptation. Gouvernement du Canada; 2014. P. 191232. https://www.rncan.gc.ca/sites/www.nrcan.gc.ca/ files/earthsciences/pdf/assess/2014/pdf/Chapitre7-Santehumaine_Fra.pdf

11. Cunsolo Willox A, Harper SL, Ford JD, Landman K, Houle K, Edge VL; Rigolet Inuit Community Government. "From this place and of this place:" climate change, sense of place, and health in Nunatsiavut, Canada. Soc Sci Med 2012 Aug;75(3):538-47. DOl PubMed
12. Dover GP, Gould R, Hayes J, Kenny G, Moore K, Mowat D, Nobbe S, Payne L, Petrucka P. Lignes directrices à l'intention des travailleurs de la santé pendant les périodes de chaleur accablante : Un guide technique, 2018. 2018-08-07. No de catalogue : H128-1/11-642F. https://www.canada.ca/fr/ sante-canada/services/sante-environnement-milieu-travail/ rapports-publications/changement-climatique-sante/lignesdirectrices-intention-travailleurs-sante-pendant-periodeschaleur-accablante-guide-technique.html

13. Demers I, Gosselin P. Pollens, climat et allergies : initiatives menées au Québec Promotion de la santé et prévention des maladies chroniques au Canada 2019;39 (4) 149-54. DOI

14. Henderson SB, Johnston FH. Measures of forest fire smoke exposure and their associations with respiratory health outcomes. Curr Opin Allergy Clin Immunol 2012 Jun;12(3):221-7. DOI PubMed

15. Health A. Impact of Wildfires on the Mental Health of Fort McMurray Residents: Neurotic Disorders, Daily Physician Visits within an Emergency Department 2015 vs. 2016. Alberta Health, Health Standards, Quality and Performance Division, Analytics and Performance Reporting Branch, 2016.

16. Cunsolo A, Ellis N. Ecological grief as a mental health response to climate change-related loss. Nat Clim Chang 2018;8(4):275-81. DOI

17. Bouchard C, Dibernardo A, Koffi J, Wood H, Leighton PA, Lindsay LR. Augmentation du risque de maladies transmises par des tiques en raison des changements climatiques et environnementaux. Relevé des maladies transmissibles au Canada 2019 45(4):89-98. DOI

18. Ludwig A, Zheng H, Vrbova L, Drebot MA, Iranpour $M$, Lindsay LR. Augmentation du risque de maladies endémiques transmises par des moustiques au Canada en raison du changement climatique. Relevé des maladies transmissibles au Canada 2019;45(4):99-107. DOI

19. Ng $V$, Rees EE, Lindsay LR, Drebot MA, Brownstone T, Sadeghieh T, Khan SU. Les changements climatiques pourraient-ils entraîner la propagation de maladies exotiques transmises par les moustiques au Canada? Relevé des maladies transmissibles au Canada 2019;45(4):108-18. DOI

20. Smith BA, Fazil A. Quelles seront les répercussions des changements climatiques sur les maladies microbiennes d'origine alimentaire au Canada? Relevé des maladies transmissibles au Canada 2019;45(4):119-25. DOI

21. Myers T, Nisbet M, Maibach E, Leiserowitz A. A public health frame arouses hopeful emotions about climate change. Clim Change 2012;113(3-4):1105-12. DOI

22. Firefighters and Nurses Top List of Canada's Most Trusted Professionals. March 15, 2018. Insights West. https:// insightswest.com/news/firefighters-and-nurses-top-list-ofcanadas-respected-professionals/

23. Watts N, Amann M, Arnell N, Ayeb-Karlsson S, Belesova K, Berry H, Bouley T, Boykoff M, Byass P, Cai W, Campbell-Lendrum D, Chambers J, Daly M, Dasandi N, 
Davies M, Depoux A, Dominguez-Salas $P$, Drummond $P$, Ebi KL, Ekins P, Montoya LF, Fischer H, Georgeson L, Grace D, Graham H, Hamilton I, Hartinger S, Hess J, Kelman I, Kiesewetter G, Kjellstrom T, Kniveton D, Lemke B, Liang L, Lott M, Lowe R, Sewe MO, Martinez-Urtaza J, Maslin M, McAllister L, Mikhaylov SJ, Milner J, Moradi-Lakeh M, Morrissey K, Murray K, Nilsson M, Neville T, Oreszczyn T, Owfi F, Pearman O, Pencheon D, Pye S, Rabbaniha M, Robinson E, Rocklöv J, Saxer O, Schütte S, Semenza JC, Shumake-Guillemot J, Steinbach R, Tabatabaei M, Tomei J, Trinanes J, Wheeler N, Wilkinson P, Gong P, Montgomery $\mathrm{H}$, Costello A. The 2018 report of the Lancet Countdown on health and climate change: shaping the health of nations for centuries to come. Lancet 2018 Dec;392(10163):2479-514. DOI PubMed

24. World Health Organization. Draft WHO global strategy on health, environment and climate change. 2018. http://www. euro.who.int/_data/assets/pdf_file/0003/378903/68id07e_ GlobalStrategyHealthEnvironmentClimateChange_180547. pdf?ua=1

25. Global Center on Adaptation. Global Commission on Adaptation. https://gca.org/global-commission-onadaptation

26. The Lancet Planetary Health. Power to the children. Lancet Planet Health 2019 Mar;3(3):e102. DOl PubMed

27. Youth climate change strikers. Open letter to world leaders. The Guardian March 1, 2019. www.theguardian.com/ environment/2019/mar/01/youth-climate-change-strikersopen-letter-to-world-leaders

28. Stevenson V. Tens of thousands rally in Montreal as part of international "school strike" against climate change. CBC News March 15, 2019. https://www.cbc.ca/news/canada/ montreal/climate-march-montreal-1.5058083

29. Appel à l'Action concernant le Changement Climatique et la Santé : des professionnels de la santé du Canada aux partis politiques fédéraux du Canada. Feb 5, 2019. https:// cape.ca/wp-content/uploads/2019/02/Appel-\%C3\%A01\%E2\%80\%99Action-concernant-le-Changement-Climatiqueet-la-Sant\%C3\%A9-Feb-5-2019-FINAL.pdf

30. International Federation of Medical Students' Associations. 2020 Vision for Climate-Health in Medical Curricula 2018 (Accessed 2018-10-09). https://docs.google.com/forms/d/e/ 1FAlpQLSeMxig6Yhs4qJU8oboKXmOKqGXRj64fcso809lHBik NGX5RYA/viewform

31. Gouvernement du Canada. Résumé de l'étude d'impact de la réglementation: Règlement modifiant le Règlement sur la réduction des émissions de dioxyde de carbone secteur de l'électricité thermique au charbon. Ministère de l'Environnement et Ministère de la Santé, 17 février 2018. http://www.gazette.gc.ca/rp-pr/p1/2018/2018-02-17/html/ reg3-fra.html
32. Government of the United Kingdom. Powering Past Coal Alliance: Partners. 2018. https://www.gov.uk/government/ publications/powering-past-coal-alliance-declaration/ powering-past-coal-alliance-partners

33. Santé Canada. Guide alimentaire canadien. 2019. https:// guide-alimentaire.canada.ca/fr/

34. Willett W, Rockström J, Loken B, Springmann M, Lang T, Vermeulen S, Garnett T, Tilman D, DeClerck F, Wood A, Jonell M, Clark M, Gordon LJ, Fanzo J, Hawkes C, Zurayk R, Rivera JA, De Vries W, Majele Sibanda L, Afshin A, Chaudhary A, Herrero M, Agustina R, Branca F, Lartey A, Fan S, Crona B, Fox E, Bignet V, Troell M, Lindahl T, Singh S, Cornell SE, Srinath Reddy K, Narain S, Nishtar S, Murray CJL. Food in the Anthropocene: the EAT-Lancet Commission on healthy diets from sustainable food systems. Lancet 2019 Feb;393(10170):447-92. DOI PubMed

35. Celis-Morales CA, Lyall DM, Welsh P, Anderson J, Steell L, Guo Y, Maldonado R, Mackay DF, Pell JP, Sattar N, Gill JMR. Association between active commuting and incident cardiovascular disease, cancer, and mortality: prospective cohort study. BMJ 2017 Apr;357:j1456. DOI PubMed

36. City of Vancouver Greenest City Action Plan. https:// vancouver.ca/files/cov/Greenest-city-action-plan.pdf

37. Rees EE, Ng V, Gachon P, Mawudeku A, McKenney D, Pedlar J, Yemshanov D, Parmely J, Knox J. Stratégie d'évaluation des risques servant à la détection précoce et à la prédiction des éclosions de maladies infectieuses associées aux changements climatiques. Relevé des maladies transmissibles au Canada 2019;45(4):132-40. DOI

38. Ogden $\mathrm{NH}$, Lindsay LR, Ludwig A, Morse AP, Zheng $H$, Zhu $H$. Prédictions d'éclosions de maladies transmises par les moustiques fondées sur les pronostics météorologiques au Canada. Relevé des maladies transmissibles au Canada 2019;45(5):141-7. DOI

39. Kotchi SO, Bouchard C, Ludwig A, Rees EE, Brazeau S. Utilisation des images d'observation de la terre pour améliorer la cartographie des risques de maladies associées au changement climatiques. Relevé des maladies transmissibles au Canada, 2019;45(5):148-58. DOI

40. Germain G, Simon A, Arsenault J, Baron G, Bouchard C, Chaumont D, El Allaki F, Kimpton A, Lévesque B, Massé A, Mercier M, Ogden NH, Picard I, Ravel A, Rocheleau JP, Soto J,pour l'Observatoire multipartite québecois sur les zoonoses et l'adaptation aux changements climatiques. Relevé des maladies transmissibles au Canada 2019;45(5):159-64. DOI

41. Ipsos Game Changers. Three Quarters (75\%) Say Canada Needs to Do More to Address Climate Change. https://www. ipsos.com/en-ca/news-polls/three-quarters-75-percent-saycanada-needs-to-do-more-to-address-climate-change 